\title{
Short communication: Milk basic protein promotes proliferation and inhibits differentiation of mouse chondrogenic ATDC5 cells
}

\author{
Sachie Nakatani, ${ }^{1}$ Yousuke Taguchi, ${ }^{1}$ Hiroya Ueda, ${ }^{1}$ Yuko Ishida, ${ }^{2}$ Yoshikazu Morita, ${ }^{2}$ Ken Kato, ${ }^{2}$ \\ Masahiro Wada, ${ }^{1}$ and Kenji Kobata ${ }^{1 *}$ \\ ${ }^{1}$ Graduate School of Pharmaceutical Sciences, Josai University, Saitama 350-0295, Japan \\ ${ }^{2}$ Milk Science Research Institute, Megmilk Snow Brand Co. Ltd., 1-1-2 Minamidai, Kawagoe, Saitama 350-1165, Japan
}

\section{ABSTRACT}

It has been reported that the intake of milk basic protein (MBP) increases bone density by promoting bone formation and suppressing bone resorption. However, few studies have been done on MBP in cartilage, the tissue adjacent to bone. We therefore investigated the effect of MBP on a chondrocyte cell line, ATDC5. In a proliferative assay using the WST-1 method, the addition of 10,100 , and $1,000 \mu \mathrm{g} / \mathrm{mL}$ of MBP to ATDC5 cells significantly increased the cell number by about 1.2-, 1.5-, and 1.7-fold, respectively, compared with the control cells. The cell cycle analysis using flow-cytometry revealed that the proportion of S- and $\mathrm{G}_{2} /$ M-phase cells was increased but that of $\mathrm{G}_{0} / \mathrm{G}_{1}$ phase was decreased in a dose-dependent manner with MBP addition. We measured the alkaline phosphatase activity of MBP-treated ATDC5 cells to examine the differentiation stage of the cells. Alkaline phosphatase activity was suppressed in a dose-dependent manner with MBP addition and was especially drastic at higher doses of MBP (100 and 1,000 $\mu \mathrm{g} / \mathrm{mL}$ ). The Alizarin Red S staining intensity, the indicator for calcification of cells, was lower in the MBP-treated $(100 \mu \mathrm{g} / \mathrm{mL})$ cells than in nontreated control cells. In the reversetranscription PCR experiment, the mRNA level of SRY-box containing gene 9 (Sox 9$)$ and type II collagen (Col2) was significantly increased in the MBP-treated cells compared with the control cells. A significant decrease of the mRNA level of runt-related transcription factor 2 (Runx2) and type X collagen (Col10) was also observed in the MBP-treated cells. These results suggested that MBP promoted the proliferation of chondrocytes by suppressing their differentiation toward calcification.

Key words: milk basic protein, chondrocyte, proliferation, differentiation gene

Received September 6, 2018.

Accepted December 7, 2018.

*Corresponding author: kobata@josai.ac.jp

\section{Short Communication}

Milk basic protein (MBP), a basic protein fraction in milk, is contained in whey protein (Kruger et al., 2007). In vitro experiments have reported that basic protein fraction of whey protein promotes the proliferation of osteoblasts and suppresses the differentiation of osteoclasts (Takada et al., 1996, 1997). In vivo experiments showed that MBP prevented bone loss in postmenopausal osteoporosis as a result of suppressing osteoclast-mediated bone resorption (Toba et al., 2000). Some clinical studies showed MBP supplementation also prevented bone loss in healthy women (Aoe et al., 2001, 2005). Another study showed MBP supplementation enhanced fracture healing in mice (Yoneme et al., 2015). It is well known that chondrocytes aggregate and differentiate in fracture sites before replacement of bone. However, few studies have evaluated the effect of MBP on cartilage tissues.

Joint epiphyses are covered with cartilage that possesses high shock absorbability, elasticity, and lubricity for the smooth movement of the joints (Scott, 2003). Chondrocytes, present in cartilage tissues, provide articular cartilage tissues with glycosaminoglycan, hyaluronic acid, and collagen (Blair et al., 2002). Chondrocytes differentiate from undifferentiated mesenchymal cells into proliferating chondrocytes, mature chondrocytes, hypertrophic chondrocytes, calcified chondrocytes, and finally into bone cells. This process of differentiation is called intra-cartilage ossification (Cancedda et al., 2000).

In this study, we investigated the effect of MBP on the proliferation and differentiation of chondrocytes, as well as the differentiation-related genes in chondrocytes. A murine chondrocyte progenitor cell line, ATDC5, was used as the model reproducing intra-cartilage ossification such as differentiation from proliferative chondrocytes to calcified chondrocytes (Atsumi et al., 1990).

First, we investigated the proliferative effect of MBP at the proliferative stage of ATDC5 using the WST-1 method. The MBP was obtained from Megmilk Snow Brand Co. Ltd. (Tokyo, Japan). The major compo- 
nents of MBP were lactoferrin (53.6\%), lactoperoxidase (36.1\%), angiogenin (3.6\%), and cystatin C (0.05\%; Ono-Ohmachi et al., 2018). The ATDC5 cells (Riken Cell Bank, Ibaraki, Japan) were cultured in a 1:1 mixture of Dulbecco's modified Eagle's medium and Ham's F12 medium, supplemented with $5 \%$ fetal bovine serum (Nichirei, Tokyo, Japan), penicillin (50 IU/mL), streptomycin $(50 \mu \mathrm{g} / \mathrm{mL})$, and kanamycin $(50 \mu \mathrm{L} / \mathrm{mL})$, and maintained at $37^{\circ} \mathrm{C}$ in a humidified atmosphere of $5 \%$ $\mathrm{CO}_{2} / 95 \%$ air. The cells were seeded at $1.5 \times 10^{3}$ cells/ well in a 96-well micro-plate, and after $18 \mathrm{~h}, \mathrm{MBP}(1$, 10,100 , and $1,000 \mu \mathrm{g} / \mathrm{mL}$ ) was added to these wells. Forty-eight hours after the addition of MBP, the culture medium was exchanged with a medium containing 10\% WST-1 reagent (Roche Diagnostics, Basel, Switzerland). After incubation for $5 \mathrm{~h}$, the absorbance of the medium at $440 \mathrm{~nm}$ was measured using the Spectra Max M2e (Nippon Molecular Devices, Tokyo, Japan). We found that the addition of 10,100 , and $1,000 \mu \mathrm{g} /$ $\mathrm{mL}$ of MBP significantly increased the cell number by about 1.2-, 1.5-, and 1.7-fold, respectively, compared with the control group (Figure 1A). Thus, the addition of MBP could significantly increase the proliferation of chondrocytes.

Next, we investigated the mechanism behind this effect by performing cell cycle analysis using flow cytometry. Eighteen hours after seeding ATDC5 cells at $1.3 \times 10^{5}$ cells/dish in a 6 -cm dish, MBP (100 and $1,000 \mu \mathrm{g} / \mathrm{mL}$ ) was added. After $24 \mathrm{~h}$ of incubation, a cell suspension was prepared using PBS $(-)$ and $0.25 \%$ trypsin-ethylenediamine tetraacetic acid solution. After removing the supernatant by centrifugation, $70 \%$ ethanol was added, and the resultant suspension was stored at $-30^{\circ} \mathrm{C}$. The stored suspension was again centrifuged to remove the supernatant. The residual cells were prepared using the Muse Cell Cycle Kit (Merck Millipore, Billerica, MA) and 5,000 of these cells were analyzed using the Muse Cell Analyzer (Merck Millipore). The effect of MBP on the cell cycle of the proliferative chondrocyte stage of ATDC5 cells is shown in Figures 1B and $1 \mathrm{C}$. In the control group, $62 \%$ of the cells were in the $\mathrm{G}_{0} / \mathrm{G}_{1}$ phase, $16 \%$ in the $\mathrm{S}$ phase, and $22 \%$ in the $\mathrm{G}_{2} / \mathrm{M}$ phase. In the group with $100 \mu \mathrm{g} / \mathrm{mL}$ of MBP, the percentage of cells in the $G_{0} / G_{1}$ phase was decreased by about $3 \%$, whereas those in the $\mathrm{S}$ phase and $\mathrm{G}_{2} / \mathrm{M}$ phase were increased by about 3 and $1 \%$, respectively, compared with the control group. In the group with $1,000 \mu \mathrm{g} / \mathrm{mL}$ of MBP, the percentage of cells in $\mathrm{G}_{0} /$ $\mathrm{G}_{1}$ phase was significantly decreased by $14 \%$, whereas those in the $\mathrm{S}$ phase and $\mathrm{G}_{2} / \mathrm{M}$ phase increased by about 6 and $8 \%$, respectively.

Alkaline phosphatase (ALP) activity is known to increase before the calcification of chondrocytes (Fedde et al., 1999; Balcerzak et al., 2003). Therefore, it could be used as an index of differentiation into hypertrophied chondrocytes. We measured the ALP activity of MBPtreated ATDC5 cells to examine the early differentiation stage of the cells. Twenty-four hours after seeding the cells at $3 \times 10^{3}$ cells/well in a 96-well micro-plate, MBP $(1,10,100$, and $1,000 \mu \mathrm{g} / \mathrm{mL})$ was added. Four days after the addition of MBP, ALP activity staining was performed. An ALP staining solution was prepared by dissolving naphthol AS-BI-phosphate (Sigma Aldrich, St. Louis, MO) and Fast Red Violet LB salt (Sigma Aldrich) in a $0.05 \mathrm{M}$ solution of 2-amino-2-methyl-1,3propanediol ( $\mathrm{pH}$ 9.8). After fixing the cells with a $20 \%$ formalin solution for $20 \mathrm{~min}$ and rinsing with water, the ALP staining solution was added, and this solution was incubated for $20 \mathrm{~min}$ at $37^{\circ} \mathrm{C}$. Dye photographs were captured using a scanner and quantified with the image processing software ImageJ (National Institutes of Health, Bethesda, MD). The ALP activity of the chondrocytes was found to be suppressed by the addition of MBP in a concentration-dependent manner (Figure 2A). With higher concentrations of MBP (100 and $1,000 \mu \mathrm{g} / \mathrm{mL}$ ), the ALP activity was drastically decreased to about one-tenth or less of the control group.

In hypertrophic chondrocytes, the production of extracellular matrix-degrading enzymes is increased, and type X collagen (Col10) induces the differentiation of the cells into calcified chondrocytes through apoptosis and calcium deposition (Cancedda et al., 2000). We prepared a staining solution by adjusting the concentration of Alizarin Red S (Sigma Aldrich) to 1\% (wt/vol) using aqueous ammonia and pure water. The ATDC5 cells, which were cultured with MBP $(100 \mu \mathrm{g} / \mathrm{mL})$ for $13 \mathrm{~d}$ in an ITS $(5 \mu \mathrm{g} / \mathrm{mL}$ of insulin, $5 \mu \mathrm{g} / \mathrm{mL}$ of transferrin, $5 \mathrm{ng} / \mathrm{mL}$ of sodium selenite)-added medium as inducer agents, was fixed with $20 \%$ formalin for $10 \mathrm{~min}$ and washed thrice with water. The staining solution was added to the cells and incubated overnight at room temperature. Dye photographs were captured with a scanner and quantified using ImageJ. We found that addition of MBP tended to suppress the mineralization of the ATDC5 cells (Figure 2B).

Transcription factors such as the sex determination region Y box 9 (Sox9), Sox5, Sox6, and runtrelated transcription factor 2 (Runx2) are known to be involved in the regulation of differentiation of chondrocytes. The Sox9 promotes the early stage and suppresses the late stage of differentiation (Ikeda et al., 2005). The Runx 2 promotes the late stage of the differentiation, transforming cells into hypertrophic chondrocytes and calcified chondrocytes (Zhang et al., 2015). In this study, we performed reverse-transcription PCR to examine the changes in the expression of differentiation-related genes in MBP-treated ATDC5 cells. For extracting a sufficient amount of RNA, 


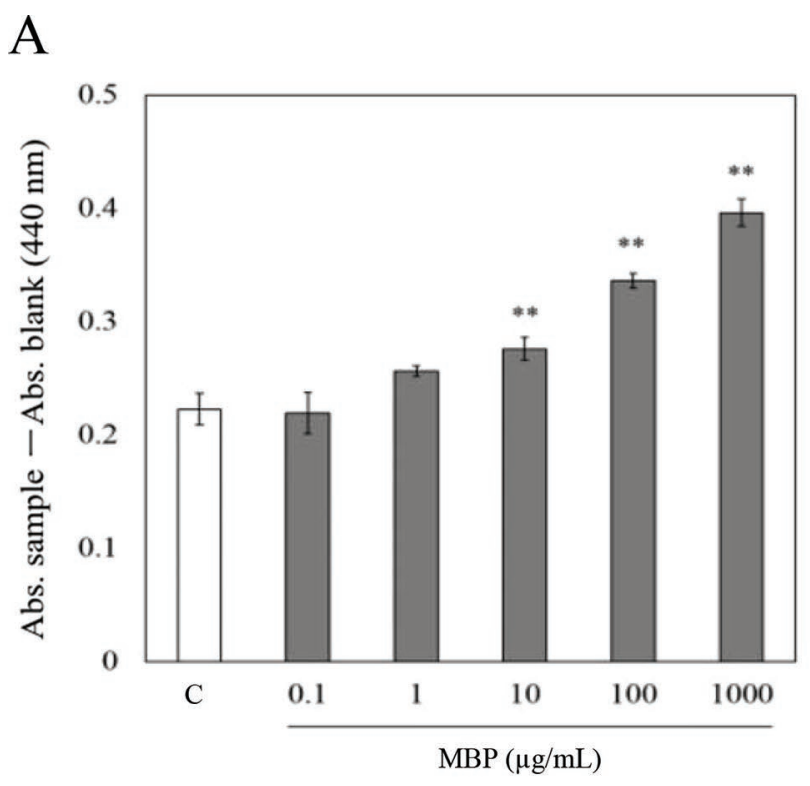

B
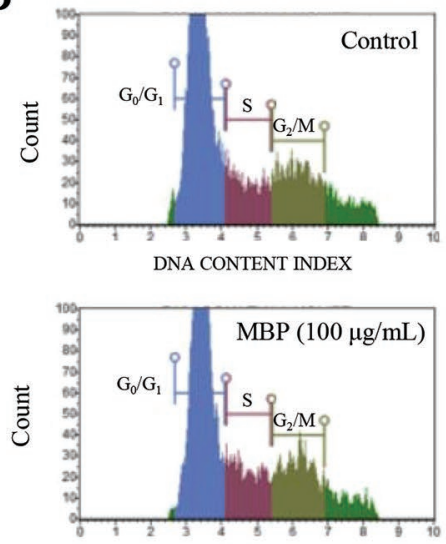

DNA CONTENT INDEX

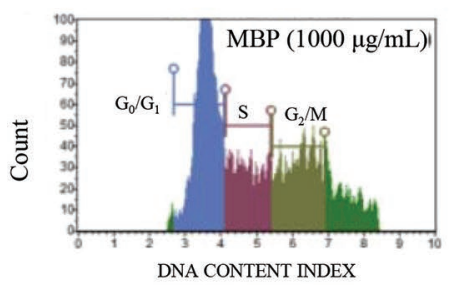

$\mathrm{C}$

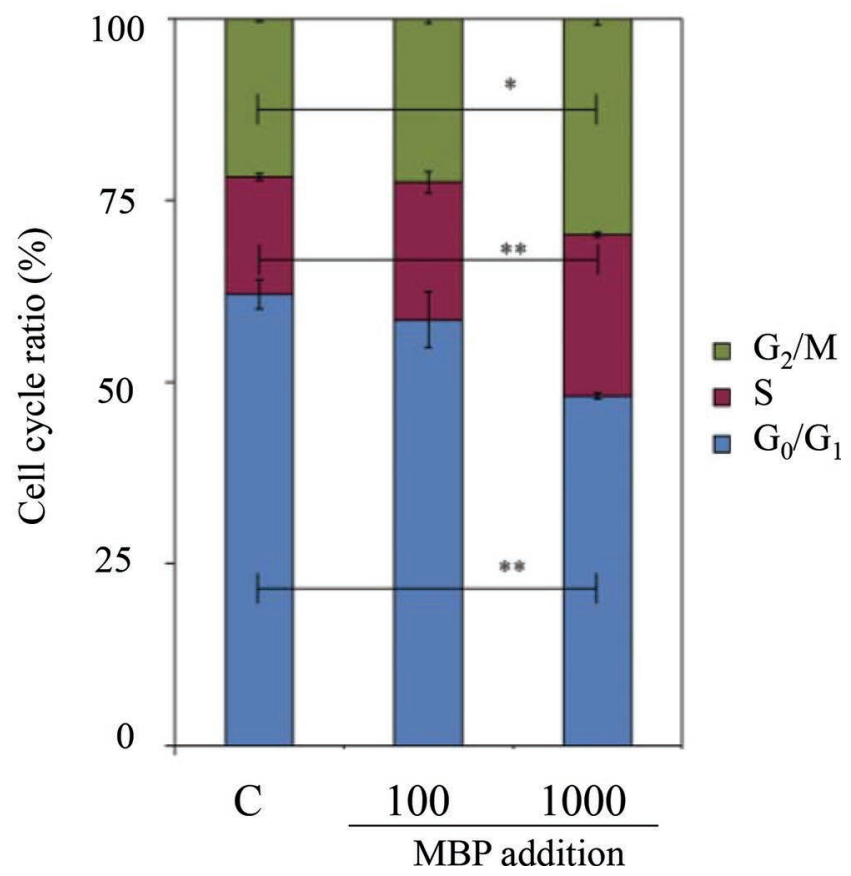

Figure 1. Effect of addition of milk basic protein (MBP) on the proliferation and cell cycle ratio of ATDC5 cells. (A) Cell proliferation (measured as absorbance, Abs.), (B) DNA content index histogram, and (C) percentage value. $\mathrm{C}=$ control. Data are presented as the mean \pm SE. Dunnett's test versus control: ${ }^{*} P<0.05 ;{ }^{* *} P<0.01(\mathrm{n}=3-5)$.

ATDC5 cells were seeded at a density of $2.0 \times 10^{5}$ cells/dish on 60-mm dishes. The day when confluence was achieved was considered d 0, and the RNA was extracted using Maxwell RSC and LEV simplyRNA Cells Kit (Promega Corporation, Madison, WI) on d 0, $3,7,14$, and 21 . The cDNA was prepared from $1 \mu \mathrm{g}$ of the total RNA of each sample by adding Oligo (dt) and reverse transcriptase (SuperScript III, Thermo Fisher Scientific, Waltham, MA), and then using the Veriti 96 well Thermal Cycler (Applied Biosystems, Foster City, CA). The PCR primers were as follows: for Gapdh, 5'-TTGACCTCAACTACATGG-3' (forward) and 5'-ATGAGGTCCACCACCCTG-3' (reverse); for Sox 9 , 5'-TGCAGCACAAGAAAGACCAC-3' (forward) and 

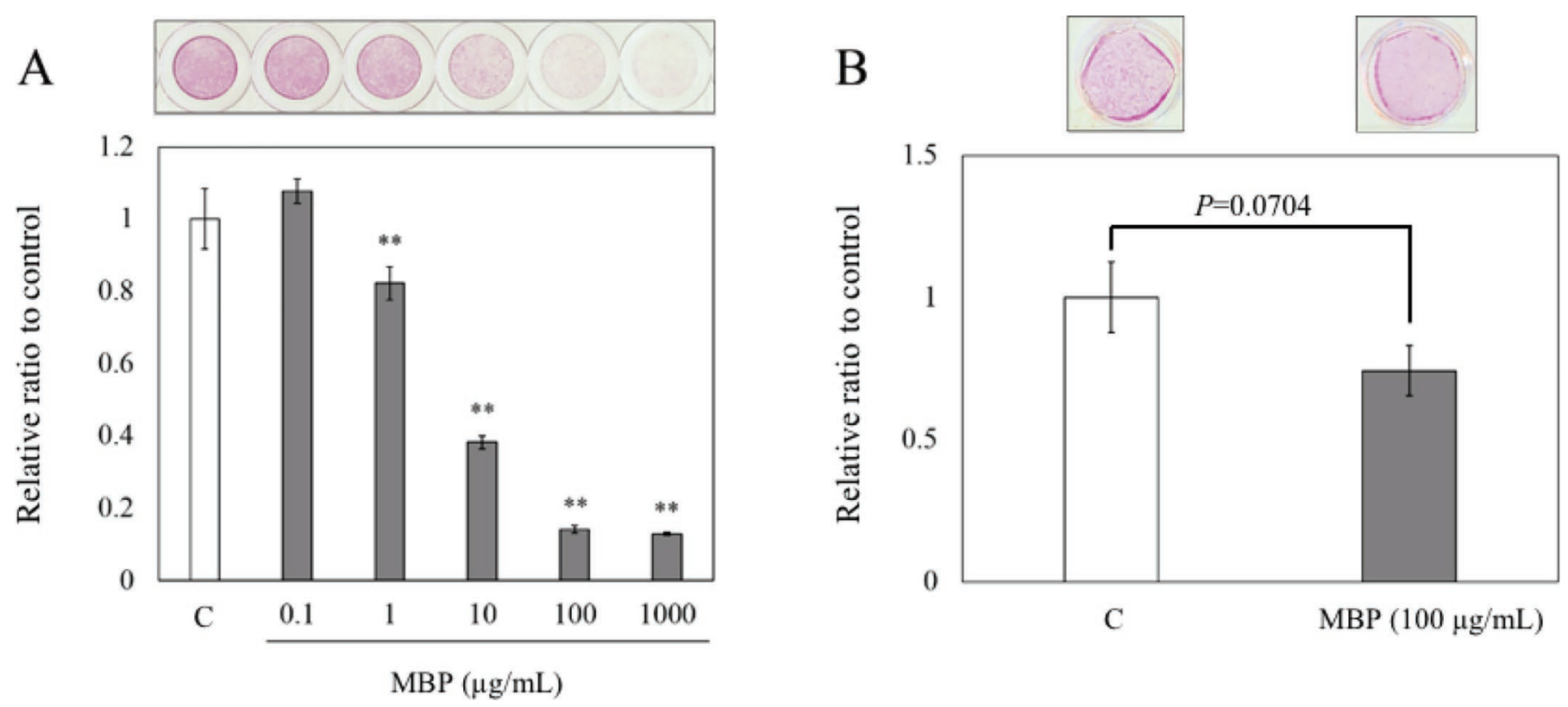

Figure 2. Effect of milk basic protein (MBP) on (A) alkaline phosphatase (ALP) activity and (B) mineralization of ATDC5 cells. Data are presented as the mean \pm SE. Dunnett's test or Student's $t$-test versus control $(\mathrm{C}):{ }^{* *} P<0.01(\mathrm{n}=4)$.

5'-CCCTCTCGCTTCAGATCAAC-3' (reverse); for Runx2, 5'-GCACTACCCAGCCACCTTTACC-3' (forward) and 5'-GTCAGCGTCAACACCATCATTC-3' (reverse); for type II collagen (Col2), 5'-GTGGAGCAGCAAGAGCAAGGA-3' (forward) and 5'-CTTGCCCCACTTACCAGTGTG-3' (reverse); and for Col10, 5'-CCACCTGGGTTAGATGGAAAA-3' (forward) and 5'-AATCTCATCAAATGGGATGGG-3' (reverse). The PCR product was electrophoresed on a 2.0\%-agarose gel at $50 \mathrm{~V}$ for $10 \mathrm{~min}$, switched to $100 \mathrm{~V}$ for $20 \mathrm{~min}$, and then stained with $5 \%$ ethidium bromide solution. The products of the electrophoresis were visualized under UV irradiation using Gel Doc EZ Imager (Applied Biosystems) and analyzed with the Image Lab software (Bio-Rad Laboratories, Hercules, CA). The expression levels of Sox9, Runx2, Col2, and Col10 mRNA were compared with that of Gapdh. As shown in Figure 3, the mRNA level of Sox9 was significantly increased on $\mathrm{d} 7$ in the MBP-added group, compared with the control group. The mRNA level of Col2 as an early marker of differentiation of cartilage also increased remarkably in the MBP-added group compared with the control group on d 3, 7, 14, and 21 . On the other hand, the mRNA level of Runx2 in the MBP-added group was lower than that of control on $d$ 7,14 , and 21, and especially on d 3 . The mRNA level of Col10, a calcified chondrocyte marker, also decreased significantly in the MBP-added group compared with the control group on $\mathrm{d} 3$.
The results of our study shed light on the effects of MBP on chondrocytes. We found that the proliferation of chondrocytes was promoted by MBP treatment. The cell cycle was likely accelerated by MBP, so more cells were probably arrested at the $\mathrm{S}$ and $\mathrm{G}_{2} / \mathrm{M}$ phases. Although MBP promoted the proliferation of chondrocytes, the mRNA levels of $5 o x 9$ and Col2 were increased by MBP. These results mean that MBP promotes the proliferation of proliferative chondrocytes and induces the differentiation from proliferative chondrocytes to mature ones. Furthermore, we found that the addition of MBP suppressed the mRNA expression of Runx2 and Col10 and decreased ALP activity and mineralization. The differentiation of chondrocytes is known to progress initially with an increase in the ALP activity until the mineralization stage is reached (Balcerzak et al., 2003). We speculated that the differentiation was delayed because it was indirectly affected by a prolonged growth phase (Figure 4). Thus, we showed that MBP promoted proliferation by affecting the cell cycle of chondrocytes. Further studies are necessary to clarify the mechanism and component behind this action of MBP on chondrocytes and to investigate the effect of MBP on chondrocytes in vivo.

\section{ACKNOWLEDGMENTS}

A part of this study was funded by Megamilk Snow Brand Co. Ltd. (Tokyo, Japan). 

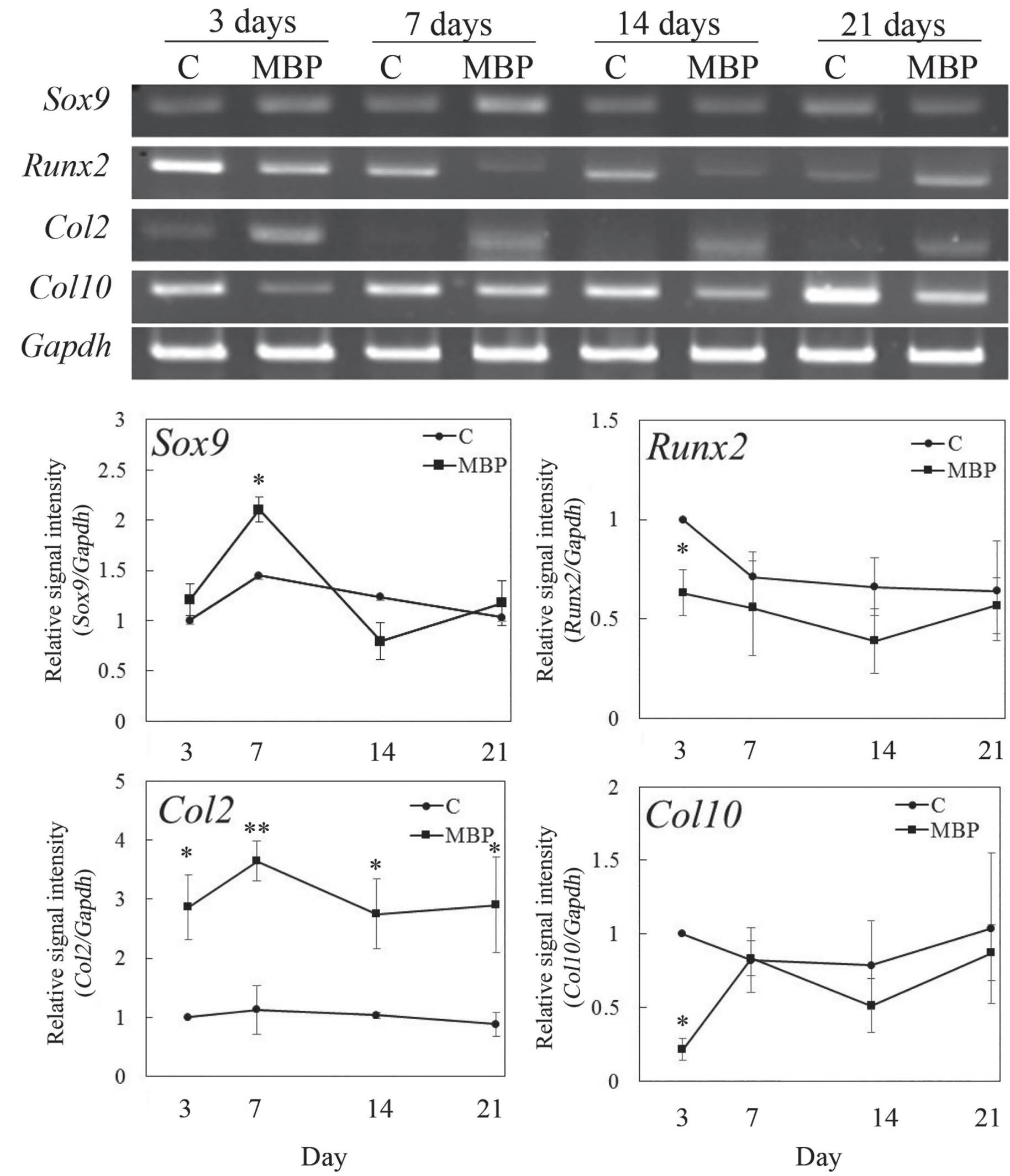

Figure 3. Effect of milk basic protein (MBP) on the expression of genes related to differentiation of ATDC5 cells. Expression levels of Sox 9 , Runx2, Col2, and Col10 are indicated relative to that of Gapdh. Data are presented as the mean \pm SE. Student's $t$-test versus control (C): ${ }^{*} P$ $<0.05 ;{ }^{* *} P<0.01(\mathrm{n}=3)$. 


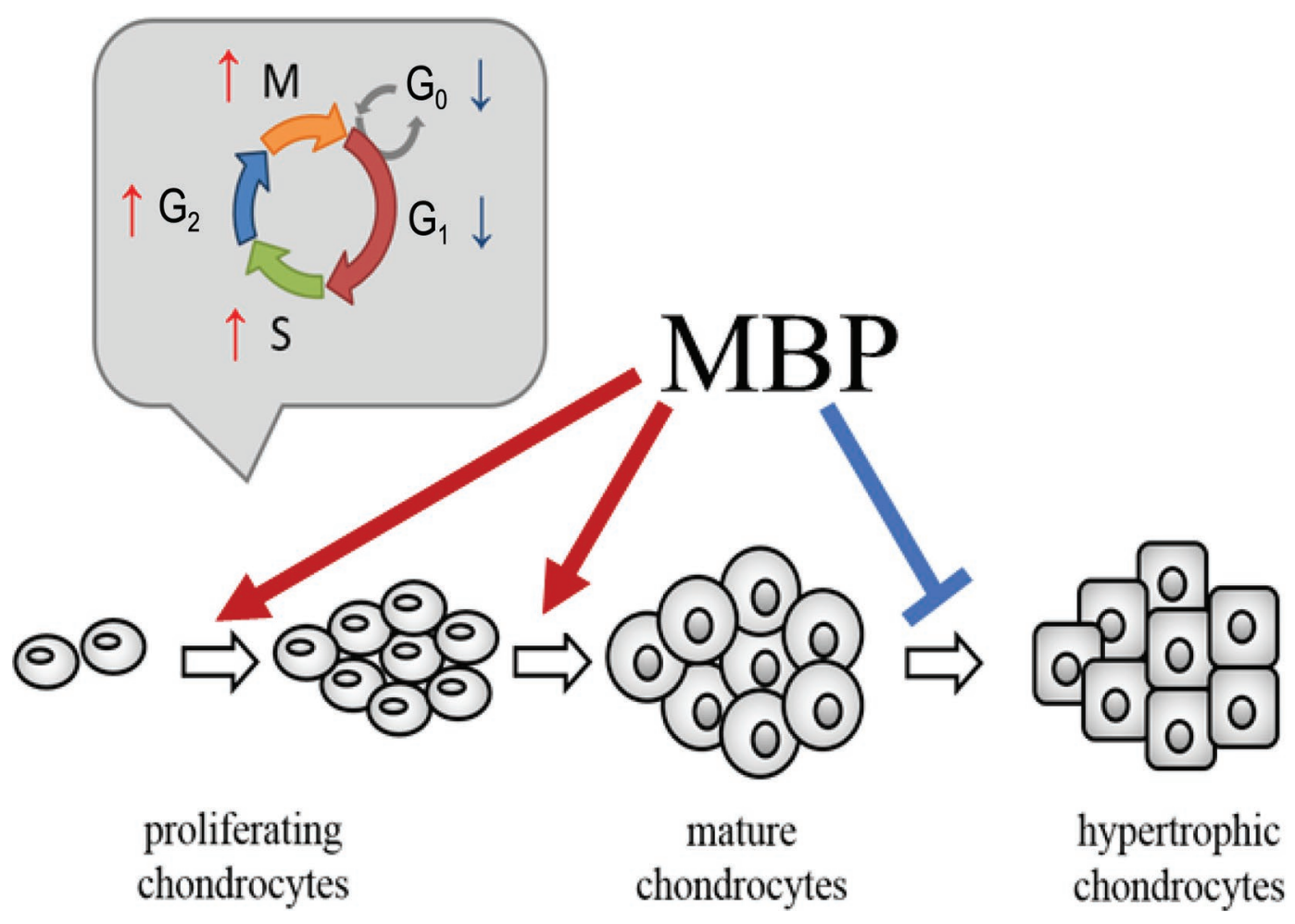

Figure 4. Schematic diagram of the effect of milk basic protein (MBP) on chondrocytes.

\section{REFERENCES}

Aoe, S., T. Koyama, Y. Toba, A. Itabashi, and Y. Takada. 2005. A controlled trial of the effect of milk basic protein (MBP) supplementation on bone metabolism in healthy menopausal women. Osteoporos. Int. 16:2123-2128.

Aoe, S., Y. Toba, J. Yamamura, H. Kawakami, M. Yahiro, M. Kumegawa, A. Itabashi, and Y. Takada. 2001. Controlled trial of the effects of milk basic protein (MBP) supplementation on bone metabolism in healthy adult women. Biosci. Biotechnol. Biochem. 65:913-918.

Atsumi, T., Y. Ikawa, Y. Miwa, and K. Kimata. 1990. A chondrogenic cell line derived from a differentiating culture of AT805 teratocarcinoma cells. Cell Differ. Dev. 30:109-116.

Balcerzak, M., E. Hamade, L. Zhang, S. Pikula, G. Azzar, J. Radisson, J. Bandorowicz-Pikula, and R. Buchet. 2003. The roles of annexins and alkaline phosphatase in mineralization process. Acta Biochim. Pol. 50:1019-1038.

Blair, H. C., M. Zaidi, and P. H. Schlesinger. 2002. Mechanisms balancing skeletal matrix synthesis and degradation. Biochem. J. 364:329-341.

Cancedda, R., P. Castagnola, F. D. Cancedda, B. Dozin, and R. Quarto. 2000. Developmental control of chondrogenesis and osteogenesis. Int. J. Dev. Biol. 44:707-714.

Fedde, K. N., L. Blair, J. Silverstein, S. P. Coburn, L. M. Ryan, R S. Weinstein, K. Waymire, S. Narisawa, J. L. Millán, G. R. MacGregor, and M. P. Whyte. 1999. Alkaline phosphatase knock-out mice recapitulate the metabolic and skeletal defects of infantile hypophosphatasia. J. Bone Miner. Res. 14:2015-2026.

Ikeda, T., H. Kawaguchi, S. Kamekura, N. Ogata, Y. Mori, K. Nakamura, S. Ikegami, and U. I. Chung. 2005. Distinct roles of Sox5,
Sox6, and Sox9 in different stages of chondrogenic differentiation. J. Bone Miner. Metab. 23:337-340.

Kruger, C. L., K. M. Marano, Y. Morita, Y. Takada, H. Kawakami, T. Kobayashi, M. Sunaga, M. Furukawa, and K. Kawamura. 2007. Safety evaluation of a milk basic protein fraction. Food Chem. Toxicol. 45:1301-1307.

Ono-Ohmachi, A., H. Nakajima-Adachi, Y. Morita, K. Kato, and S. Hachimura. 2018. Milk basic protein supplementation exerts an anti-inflammatory effect in a food-allergic enteropathy model mouse. J. Dairy Sci. 101:1852-1863.

Scott, J. E. 2003. Elasticity in extracellular matrix 'shape modules' of tendon, cartilage, etc. A sliding proteoglycan-filament model. J. Physiol. 553:335-343.

Takada, Y., S. Aoe, and M. Kumegawa. 1996. Whey protein stimulated the proliferation and differentiation of osteoblastic MC3T3-E1 cells. Biochem. Biophys. Res. Commun. 223:445-449.

Takada, Y., N. Kobayashi, H. Matsuyama, K. Kato, J. Yamamura, M. Yahiro, M. Kumegawa, and S. Aoe. 1997. Whey protein suppresses the osteoclast-mediated bone resorption and osteoclast cell formation. Int. Dairy J. 7:821-825.

Toba, Y., Y. Takada, J. Yamamura, M. Tanaka, Y. Matsuoka, H. Kawakami, A. Itabashi, S. Aoe, and M. Kumegawa. 2000. Milk basic protein: A novel protective function of milk against osteoporosis. Bone 27:403-408.

Yoneme, H., J. Hatakeyama, A. Danjo, H. Oida, M. Yoshinari, R. Aijima, N. Murata, T. Watanabe, Y. Oki, and M. A. Kido. 2015. Milk basic protein supplementation enhances fracture healing in mice. Nutrition 31:399-405.

Zhang, Y., T. L. Yang, X. Li, and Y. Guo. 2015. Functional analyses reveal the essential role of SOX6 and RUNX2 in the communication of chondrocyte and osteoblast. Osteoporos. Int. 26:553-561. 